\title{
A CHARACTERIZATION OF SMOOTH FUNCTIONS DEFINED ON A BANACH SPACE
}

\author{
RICHARD M. HAIN
}

\begin{abstract}
A sufficient condition for a function defined on a Banach space to be $C^{k}$ is given. This enables us to characterize the $C^{\infty}$ functions from one Banach space into another Banach space as those functions that, for each positive integer $m$, have the property that the composition of the function with each $C^{\infty}$ function from $\mathbf{R}^{m}$ into the domain of the function is $C^{\infty}$.
\end{abstract}

1. Introduction. Due to the nature of the definition of the higher Fréchet derivatives of a function defined on a Banach space it is sometimes difficult to determine whether a given function is of class $C^{k}$. In this paper we give a sufficient condition for a function defined on a Banach space to be $C^{k}$ $(1 \leqslant k<\infty)$. As a corollary we characterize the $C^{\infty}$ functions from one Banach space into another Banach space as those functions that, for each positive integer $m$, have the property that the composition of the function with each $C^{\infty}$ function from $\mathbf{R}^{m}$ into the domain of the function is $C^{\infty}$.

Recall the following facts from the calculus of Banach spaces. This material may be found in [5] or [6]. Let $E$ and $F$ be Banach spaces, $\Omega$ an open subset of $E$ and $f: \Omega \rightarrow E$ a function of class $C^{k}$ where $k$ is a positive integer. For each integer $p$, between 0 and $k$, the $p$ th derivative $D^{p} f$ of $f$ is a continuous function defined on $\Omega$ and taking values in $L^{p}(E, F)$, the Banach space of bounded $p$ multilinear functions from $E^{p}$ into $F$. For an element $\Phi$ of $L^{p}(E, F)$ the norm of $\Phi$ is given by

$$
\|\Phi\|=\sup \left\{\left\|\Phi\left(v_{1}, \ldots, v_{p}\right)\right\|: v_{j} \in E,\left\|v_{j}\right\| \leqslant 1 \text { and } 1 \leqslant j \leqslant p\right\} .
$$

\section{The Main Theorem.}

THEOREM 1. For each positive integer $k$, a function from a Banach space into another Banach space is of class $C^{k}$ if its composition with each $C^{\infty}$ function from $\mathbf{R}^{k+1}$ into the domain of the function is of class $C^{k+1}$. In particular, such a function is of class $C^{\infty}$ if and only if for all positive integers $m$ its composition with each $C^{\infty}$ function from $\mathbf{R}^{m}$ into the domain of $f$ is $C^{\infty}$.

RemarKs. (i) A more desirable version of the theorem would read: "A function between two Banach spaces is of class $C^{k}$ if and only if its composition with each $C^{\infty}$ function from $\mathbf{R}^{k+1}$ into the domain of the function is of class $C^{k}$." This is certainly true when the dimension of the

Received by the editors November 1, 1978.

AMS (MOS) subject classifications (1970). Primary 26A96, 58C20; Secondary 46B99, 49F05.

Key words and phrases. Fréchet derivative, Gateaux derivative. 
domain is finite but its truth is unknown in the general case.

(ii) Even when the domain of the function is of finite dimension it is not true that if the composition of the function with each $C^{\infty}$ path is $C^{1}$, then the function is of class $C^{1}$, as the following example shows. Let $(r, \theta)$ denote the usual polar coordinates in $\mathbf{R}^{2}$. Define $f: \mathbf{R}^{2} \rightarrow \mathbf{R}$ by $f(r, \theta)=r \sin 3 \theta$. It is easily seen that $f$ is not Fréchet differentiable at the origin although for all smooth paths $\gamma: \mathbf{R} \rightarrow \mathbf{R}^{2}$, the path $f \circ \gamma$ is of class $C^{1}$.

The following lemma is due to K. T. Chen [3] and I am grateful to him for allowing me to reproduce it here.

LEMMA 2. If $\left\{b_{n}\right\}$ is a sequence of points in a normed vector space $E$ such that $\left\|b_{n}\right\|=O\left(\exp \left(-2^{n}\right)\right)$ as $n \rightarrow \infty$, then there exists a $C^{\infty}$ path $\gamma: \mathbf{R} \rightarrow E$ such that $\gamma\left(1-2^{-n}\right)=b_{n}$ and $\gamma(1)=0$.

Proof. Choose a $C^{\infty}$ function $\theta: \mathbf{R} \rightarrow \mathbf{R}$ with the following properties; $\theta(x)=0$ whenever $x \leqslant 0$ and $\theta(x)=1$ whenever $x \geqslant 1$. For convenience set $t_{n}=1-2^{-n}$ and define $S_{n}:\left[t_{n-1}, t_{n}\right] \rightarrow[0,1]$ by $S_{n}(t)=2^{n}\left(t-t_{n}\right)$. Now define $\gamma: \mathbf{R} \rightarrow E$ by

$$
\gamma(t)= \begin{cases}b_{0}, & t \leqslant 0, \\ \theta \circ S_{n}(t) b_{n}+\left(1-\theta \circ S_{n}(t)\right) b_{n-1}, & t_{n-1} \leqslant t \leqslant t_{n}, \\ 0, & t>1 .\end{cases}
$$

Observe that if $t \in\left[t_{n-1}, t_{n}\right]$, then for each positive integer $p$

$$
\left\|\gamma^{(p)}(t)\right\|=O\left(2^{p n} \exp \left(-2^{n}\right)\right) \quad \text { as } n \rightarrow \infty \text {. }
$$

It follows from the mean value theorem that $\gamma$ is a $C^{\infty}$ path.

An interesting consequence of this lemma is the following result.

COROllary 3. A function defined on a normed vector space is continuous if and only if its composition with each $C^{\infty}$ path in the domain of the function is continuous.

We shall of ten denote a typical element $\left(v_{1}, \ldots, v_{p}\right)$ of the $p$-fold Cartesian product $E^{p}$ of $E$ with itself by $\mathbf{v}$; a typical element of the sequence $\left\{\mathbf{v}^{n}\right\}$ in $E^{p}$ being $\left(v_{1}^{n}, \ldots, v_{p}^{n}\right)$. We define a distance function on $E^{p}$ by defining the distance $d(\mathbf{v}, \mathbf{w})$ between two elements $\mathbf{v}$ and $\mathbf{w}$ of $E^{p}$ by

$$
d(\mathbf{v}, \mathbf{w})=\max \left\{\left\|v_{j}-w_{j}\right\|: 1 \leqslant j \leqslant p\right\} .
$$

As usual $\left\{e_{1}, \ldots, e_{p}\right\}$ denotes the standard basis of $\mathbf{R}^{p}$.

LEMMA 4. If $E$ is a normed vector space, $\left\{a_{n}\right\}$ a sequence in $E$ converging to $b$ and $\left\{\mathbf{w}^{n}\right\}$ a sequence in $E^{p}$ converging to $\mathbf{v}$, then there is a subsequence $\left\{b_{n}\right\}$ of $\left\{a_{n}\right\}$ and a corresponding subsequence $\left\{v^{n}\right\}$ of $\left\{\mathbf{w}^{n}\right\}$ and $a C^{\infty}$ function $\alpha$ : $\mathbf{R} \times \mathbf{R}^{p} \rightarrow E$ such that

(i) for each $t$ in $\mathbf{R}$, the function $\alpha(t,-) ; \mathbf{R}^{p} \rightarrow E$ is an affine map,

(ii) for each positive integer $n$ and each integer $j$ with $1 \leqslant j \leqslant p$ we have 
$\alpha\left(1-2^{-n}, e_{j}\right)=b_{n}+v_{j}^{n}, \alpha\left(1-2^{-n}, 0\right)=b_{n}, \alpha\left(1, e_{j}\right)=b+v_{j}$ and $\alpha(1,0)=$ $b$.

Proof. Choose a subsequence $\left\{n_{k}\right\}$ of $\{n\}$ such that the corresponding subsequences $\left\{b_{n}\right\}$ of $\left\{a_{n}\right\}$ and $\left\{\mathbf{v}^{n}\right\}$ of $\left\{\mathbf{w}^{n}\right\}$ satisfy

$$
\left\|b-b_{n}\right\|=O\left(\exp \left(-2^{n}\right)\right) \text { and }\left\|\mathbf{v}-v^{n}\right\|=O\left(\exp \left(-2^{n}\right)\right)
$$

as $n \rightarrow \infty$. Let $t_{n}, \theta: \mathbf{R} \rightarrow \mathbf{R}$ and $S_{n}:\left[t_{n-1}, t_{n}\right] \rightarrow[0,1]$ be as in the proof of Lemma 2. For an element $\mathbf{w}$ of $E^{p}$ and an element $\mathbf{x}$ of $\mathbf{R}^{p}$, denote by $\mathbf{x} \cdot \mathbf{w}$ the element $x_{1} w_{1}+\cdots+x_{p} w_{p}$ of $E$. Define $\alpha: \mathbf{R} \times \mathbf{R}^{p} \rightarrow E$ by

$$
\alpha(t, \mathbf{x})=\left\{\begin{array}{l}
b_{0}+\mathbf{x} \cdot \mathbf{v}^{0}, \quad t \leqslant 0, \\
\theta \circ S_{n}(t)\left(b_{n}+\mathbf{x} \cdot \mathbf{v}^{n}\right)+\left(1-\theta \circ S_{n}(t)\right)\left(b_{n-1}+\mathbf{x} \cdot \mathbf{v}^{n-1}\right), \\
t_{n-1} \leqslant t \leqslant t_{n}, \\
b+\mathbf{x} \cdot \mathbf{v}, \quad t \geqslant 1 .
\end{array}\right.
$$

Let $E$ and $F$ be Banach spaces and $p$ a positive integer. Denote the subspace of $L^{p}(E, F)$ consisting of the bounded symmetric $p$ multilinear functions from $E^{p}$ into $F$ by $L_{s}^{p}(E, F)$. For an element $v$ of $E$, denote the element $(v, \ldots, v)$ of $E^{p}$ by $v^{p}$. The following result is well known and will not be proved. A proof may be found in [1] or [2].

LEMMA 5. Let $E$ and $F$ be Banach spaces, $\Omega$ an open convex subset of $E, f$ an $F$ valued function defined on $\Omega$ and $\varphi_{p}: \Omega \rightarrow L_{s}^{p}(E, F)(0<p<k)$ continuous functions. Whenever $\|v\|$ is small enough we may define for each a in $\Omega$

$$
R(a, v)=f(a+v)-\sum_{p=0}^{k} \frac{\varphi_{p}(a) v^{p}}{p !}
$$

If for each element $b$ of $\Omega$ we have $\|R(a, v)\|=o\left(\|v\|^{k}\right)$ as $(a, v) \rightarrow(b, 0)$, then $f$ is of class $C^{k}$ and $D^{p} f=\varphi_{p}$ for each integer $p$ with $0<p<k$.

I am grateful to $\mathrm{H}$. Porta for showing me the following lemma from linear algebra and providing its proof.

LEMMA 6. Let $E$ and $F$ be real vector spaces, $p$ a positive integer and $f a$ symmetric function defined on $E^{p}$ and taking values in $F$. If

(i) For each element $\left(v_{1}, \ldots, v_{p}\right)$ of $E^{p}$ and each real number $\lambda$ we have

$$
f\left(\lambda v_{1}, v_{2}, \ldots, v_{p}\right)=\lambda f\left(v_{1}, v_{2}, \ldots, v_{p}\right)
$$

and

(ii) $f\left(v_{0}+v_{1}, v_{2}, \ldots, v_{p}\right)=f\left(v_{0}, v_{2}, \ldots, v_{p}\right)+f\left(v_{1}, v_{2}, \ldots, v_{p}\right)$ whenever $v_{0}, v_{1}, \ldots, v_{p}$ are linearly dependent vectors in $E$, then $f$ is a symmetric $p$ multilinear function from $E^{p}$ into $F$.

Proof. We need only consider the case when $p=2$. Suppose that $f$ : $E \times E \rightarrow F$ is a function satisfying conditions (i) and (ii) above. Let $x, y, z$ be arbitrary elements of $E$. Applying (ii) twice we see that 


$$
\begin{gathered}
f(x+y+z, x+y+z)-f(x, x)-f(y, y)-f(z, z) \\
=2 f(x+y, z)+2 f(x, y) .
\end{gathered}
$$

Since the left-hand side is symmetric in $x, y$ and $z$ we conclude that

$$
f(x+y, z)+f(x, y)=f(x+z, y)+f(x, z) .
$$

That is

$$
f(x+y, z)-f(x, z)=f(x+z, y)-f(x, y) .
$$

By replacing $y$ by $2 y$ in (2.1) and applying (2.1) again we obtain

$$
f(x+2 y, z)+f(x, z)=2 f(x+y, z) .
$$

Similarly one obtains

$$
f(2 x+y, z)+f(y, z)=2 f(x+y, z) .
$$

Now replace $x$ by $2 x$ in (2.2) and apply (2.3) to see that

$$
f(2 x+2 y, z)+2 f(x, z)=4 f(x+y, z)-2 f(x, z) .
$$

That is

$$
f(x+y, z)=f(x, z)+f(y, z) .
$$

Proof of Theorem 1. Let $E$ and $F$ be Banach spaces, $k$ a positive integer and $f: E \rightarrow F$ a function such that for each $C^{\infty} \operatorname{map} \alpha: \mathbf{R}^{k+1} \rightarrow E$, the composite $f \circ \alpha$ is of class $C^{k+1}$. Our first task is to construct for each integer $p$ between 1 and $k+1$ candidates for $D^{p} f(b)$. For a fixed point $b$ in $E$ and fixed $\mathbf{v}$ in $E^{p}$, define a $C^{\infty}$ function $\alpha: \mathbf{R}^{p} \rightarrow E$ by $\alpha(\mathbf{x})=b+x \cdot \mathbf{v}$ (the notation is as in the proof of Lemma 4). Now define $\varphi_{p}: E \rightarrow L_{s}^{p}(E, F)$ by

$$
\varphi_{p}(b)(\mathbf{v})=D^{p}(f \circ \alpha)(0)\left(e_{1}, \ldots, e_{p}\right) .
$$

Observe that the right-hand side makes sense because $f \circ \alpha$ is assumed to be of class $C^{k+1}$. Since $f \circ \alpha$ is of class $C^{p}$, it follows that for each $b$ in $E, \varphi_{p}(b)$ satisfies the conditions of Lemma 6 . We conclude that $\varphi_{p}(b)$ is a symmetric $p$ multilinear function from $E^{p}$ into $F$.

Next we show that the function $\delta^{p} f: E \times E^{p} \rightarrow F$ defined by

$$
\delta^{p} f(b, \mathbf{v})=\varphi_{p}(b)(\mathbf{v})
$$

is continuous. If $\left\{\left(b_{n}, \mathbf{v}^{n}\right)\right\}$ is a sequence in $E \times E^{p}$ converging to $(b, \mathbf{v})$, then according to Lemma 4 we can, by dropping to a subsequence if necessary, define a $C^{\infty}$ function $\alpha: \mathbf{R} \times \mathbf{R}^{p} \rightarrow E$ satisfying the conditions set out in Lemma 4. It follows from (2.4) and the fact that $f \circ \alpha$ is of class $C^{k+1}$ that

$$
\begin{aligned}
\delta^{p} f(b, \mathbf{v}) & =D^{p}(f \circ \alpha)(1,0)\left(e_{1}, \ldots, e_{p}\right) \\
& =\lim _{n \rightarrow \infty} D^{p}(f \circ \alpha)\left(1-2^{-n}, 0\right)\left(e_{1}, \ldots, e_{p}\right) \\
& =\lim _{n \rightarrow \infty} \delta^{p} f\left(b_{n}, \mathbf{v}^{n}\right) .
\end{aligned}
$$

Observe that in particular we have shown that for each $b$ in $E$, the function $\varphi_{p}(b)$ is a bounded multilinear function.

Our final task is to show that $f$ is of class $C^{k}$. According to Lemma 5 we 
need only show that $R(a, v)=O\left(\|v\|^{k}\right)$ as $(a, v) \rightarrow(b, 0)$ and that $\varphi_{p}$ is continuous for $0 \leqslant p \leqslant k$. Since the proof of Taylor's theorem depends only upon the restriction of the function to a line segment we have

$$
R(a, v)=\int_{0}^{1} \frac{(1-t)^{k-1}}{(k-1) !}\left\{\varphi_{k}(a+t v)\left(v^{k}\right)-\varphi_{k}(a)\left(v^{k}\right)\right\} d t
$$

It follows that if $\varphi_{k}$ is continuous, then $\|R(a, v)\|=o\left(\|v\|^{k}\right)$ as $(a, v) \rightarrow(b, 0)$.

To complete the proof we demonstrate the continuity of $\varphi_{p}(0 \leqslant p \leqslant k)$. We use induction. When $p=0$ the result follows from Corollary 3. Suppose that we have shown that $f$ is of class $C^{k-1}$, we will show that $\varphi_{k}$ is continuous.

If $b, u, v_{1}, \ldots, v_{k}$ are points in $E$, then

$$
\begin{aligned}
\varphi_{k}(b+u) & \left(v_{1}, \ldots, v_{k}\right)-\varphi_{k}(b)\left(v_{1}, \ldots, v_{k}\right) \\
& =\int_{0}^{1} \varphi_{k+1}(b+t u)\left(u, v_{1}, \ldots, v_{k}\right) d t
\end{aligned}
$$

Thus

$$
\begin{aligned}
\| \varphi_{k}(b+u) & \left(v_{1}, \ldots, v_{k}\right)-\varphi_{k}(b)\left(v_{1}, \ldots, v_{k}\right) \| \\
& \leqslant \sup _{t \in[0,1]}\left\|\varphi_{k+1}(b+t u)\left(u, v_{1}, \ldots, v_{k}\right)\right\| .
\end{aligned}
$$

Since $\delta^{k+1} f$ is a continuous function, for each $\varepsilon>0$ we can find $\rho>0$ such that $\rho<1$ and if $x, w_{1}, \ldots, w_{k}$ are points in $E$ all with norm less than $\rho$, then

$$
\left\|\delta^{k+1} f(b+x)\left(w_{1}, \ldots, w_{k}\right)\right\|<\varepsilon .
$$

Suppose now that for $1 \leqslant j \leqslant k$ we have $\left\|v_{j}\right\| \leqslant 1$. If $\|u\|<\rho^{k+1}$, then

$$
\left\|\delta^{k+1} f(b+t u)\left(u, v_{1}, \ldots, v_{k}\right)\right\|=\left\|\delta^{k+1} f(b+t u)\left(\rho^{-k} u, \rho v_{1}, \ldots, \rho v_{k}\right)\right\|<\varepsilon .
$$

It follows that $\varphi_{k}$ is continuous and the theorem is proved.

REMARK. As a corollary of the theorem we deduce that if we consider a Banach space $E$ as a differentiable space (in the sense of Chen [4]) where the plots are generated by the $C^{\infty}$ functions from finite dimensional Euclidean vector spaces into $E$, then the notion of smooth differentiable space 1 forms coincides with the usual notion of smooth 1 forms on a Banach space (see for example Lang [5]). This result can be applied to the calculus of variations on Banach spaces (see [3]).

\section{REFERENCES}

1. R. Abraham and J. Robbin, Transversal mappings and flows, Benjamin, New York, 1967.

2. F. Albrecht and H. Diamond, A converse of Taylor's theorem, Indiana Univ. Math. J. 21 (1971/72), 347-350.

3. K. T. Chen, Global calculus, mimeographed class notes, 1976.

4. ___ Iterated path integrals, Bull. Amer. Math. Soc. 83 (1977), 831-879.

5. S. Lang, Differentiable manifolds, Addison-Wesley, Reading, Mass., 1972.

6. J. T. Schwartz, Nonlinear functional analysis, Gordon and Breach, New York, 1969.

Department of Mathematics, University of Illinois, Urbana, ILLINOIS 61801 